\title{
Susanne Hildebrandt
}

\section{Die Privatisierung des mexikanischen Ejido"}

Der Artikel 27 der mexikanischen Verfassung garantiert das Ejido - eine spezifische Form des gemeinschaftlichen Landbesitzes bäuerlicher Gemeinden. Die 1992 erfolgte Reform dieses Artikels ist ein zentraler Baustein der neoliberalen Modernisierung auf dem Land, die Voraussetzungen für die Privatisierung des Ejidos wurden damit geschaffen. Die Änderung der Besitzverhältnisse folgte aber einem ökonomistischen Kurzschluss: die Landkonzentration wird mit der Argument gefördert, die mexikanische Landwirtschaft sei wegen der geringen Größe der Güter und hier vor allem des Ejido unproduktiv und unrentabel. Gleichzeitig mit dem Rückzug eines traditionell interventionistischen Staates aus der Landwirtschaft, sollten auch die traditionell klientelistischen Beziehungen zwischen Staat und Gesellschaft modernisiert werden. Die akkumulierten Effekte dieser Politik lösten auf dem Land heftige Proteste unter den Betroffenen aus, die sich vor allem in zwei Bewegungen manifestieren: 1) die bankrotten, privaten Landbesitzer schlossen sich ab 1993 zur nationalen Schuldnerbewegung El Barzón zusammen; 2) die bewaffnete Revolte der Zapatisten (EZLN) in Chiapas von 1994 und die Entstehung von Guerillas in weiteren Südstaaten sind Ausdruck des Protests der campesinos gegen die Bedrohung ihrer Lebensgrundlage. Im folgenden werden die Rolle des Ejido in der mexikanischen Gesellschaft und die ökonomischen wie politischen Folgen seiner Privatisierung untersucht. Schließlich wird die ganze Problematik noch an einem besonders prägnanten Beispiel, dem Ejido von Sayula illustriert.

\section{Das alte Ejido - Präkolumbianischen Ursprünge und revolutionärer Kontext}

Das Ejido als ein System des gemeinschaftlichen Landbesitzes von bäuerlichen Gemeinden - den pueblos ${ }^{1}$ - hat präkolumbianische Wurzeln. Auf dem Territorium des heutigen Mexiko geht der gemeinschaftliche Landbesitz zurück auf eine Tradition der Azteken, ist aber im ganzen Subkontinent geläu-

\footnotetext{
* Dieser Artikel beruht wesentlich auf 2 1/2 Jahren Feldforschung (1996-98) in Sayula/Jalisco im Rahmen meiner Doktorarbeit Weltmarktintegration und Legitimität des politischen Systems in Mexiko. Eine Fallstudie im ländlichen Raum: das Municipio Sayula/Jalisco, 1982-98.

1 Im Spanischen hat das Wort die Doppelbedeutung von „Volk“ und „Dorf“ bzw. „kleinere Stadt“" (als Siedlungsform).
} 
fig. Land und die Landfrage waren die zentralen Themen der mexikanischen Geschichte. Das 19. und 20. Jahrhundert hindurch haben Bauern und indigene Gemeinden für ein Stück Land zum Leben gekämpft. Während der mexikanischen Revolution 1910-17 ging es der Bauernschaft ganz wesentlich um die Wiederherstellung ihrer Landrechte, die sie durch die liberale Agrarpolitik im 19. Jahrhundert verloren hatten. Bei Ausbruch der Revolution von 1910 besaßen 92\% der Landbevölkerung kein Land. Nur 1\% der Landbesitzer kontrollierten 97\% des Ackerlands. ${ }^{2}$

Die mexikanische Verfassung von 1917 kennt drei Arten des Landbesitzes: nationales Eigentum, gemeinschaftlicher und privater Grundbesitz. Die verfassungsmäßige Verankerung des Ejido im Artikel 27 und somit die Gewährung staatlichen Schutzes war eine zentrale Errungenschaft der mexikanischen Revolution von 1910. 70 Jahre lang war die Agrarreform das wichtigste Thema der Politik; der junge Nationalstaat baute darauf seine Identität. ${ }^{3}$ Der Großteil der Ejidos wurde ab Ende der 20er ${ }^{4}$ bis Ende der 60er Jahre ${ }^{5}$ gegründet. Anfangs unter starken Kämpfen durch Enteignung von Latifundien, später auch durch Ausdehnung der Agrargrenze (durch Bewässerung oder Rodung von Urwald). Der VII Agrarzensus von 1991 wies eine Gesamtzahl von 29.951 Ejidos und comunidades agrarias ${ }^{6}$ aus, die zusammen eine Fläche von 102.876.789 Hektar ausmachen, was ca. 53\% des nationalen Territoriums entsprach. Über ein dreiviertel Jahrhundert blieben die verfassungsmäßigen Grundlagen des Landbesitzes unangetastet. Das Ejido schuf zwei Klassen un-

2 Bei der Beurteilung der mexikanischen Realität sollte man stets das Bevölkerungswachstum im Auge haben: 19009 Millionen; 191015 Mio; 194020 Mio; 197048 Mio; 198560 Mio; 200090 Millionen. Im Vergleich dazu: die deutsche Bevölkerung ist im 20. Jahrhundert nahezu konstant bei 80 Mio. geblieben.

3 Die offizielle Ideologie des agrarismo kommt in dem revolutionären Schlachtruf zum Ausdruck: „La tierra a quien la trabaja” (dt.: Das Land dem, der es bearbeitet).

4 Anfänglich ging die Landenteignung schleppend voran: 1923 besaßen 2\% der privaten Landbesitzer immer noch $82 \%$ des Ackerlands. 59\% der Landbevölkerung besaß nur 0,8\% des Ackerlands.

5 Die zweite Welle der Landenteignung unter Präsident Cárdenas (1934-40) brachte die Wende. Gegen Ende seiner Amtszeit waren 50\% des Ackerlands in Ejidobesitz. 1970 war 43\% des Ackerlands auf 66\% der Landbevölkerung verteilt worden, die alle größtenteils Ejidatarios waren.

6 Gleich von Anfang an unterscheidet die Verfassung von 1917 zwischen zwei Kategorien von Begünstigten der Agrarreform: a) die comunidades agrarias und b) die Ejidos.. Bei den comunidades agrarias handelt es sich um indianische Gemeinden, denen ihre Länder geraubt worden waren, die einen alten Besitztitel innehatten (oft noch aus Zeiten der spanischen Krone), der sie als rechtmäßige Eigentümer der beanspruchten Ländereien auswies. Es ging hier lediglich um die Wiederherstellung der alten Landrechte, die Rückgabe von geraubtem Land und die Wiedergutmachung eines erlittenen Unrechts. Bei den Ejidos handelte es sich um Neugründungen, die auf Antrag einer Gruppe von landlosen Bauern erfolgte. Die antragstellende Bauerngruppe erhielt eine vom Staatspräsidenten unterzeichnete Genehmigung zur Gründung eines Ejido. Der Staat (vertreten durch das Agrarministerium) bewerkstelligte die Enteignung von Großgrundbesitzern (mit finanzieller Entschädigung) oder sorgte für die Inwertsetzung von Brachland. 
ter der Landbevölkerung: eine Minderheit von Berechtigten (1/3) und eine Mehrheit von Nicht-Berechtigten (2/3). Mit der Gründung des nationalen Bauernverbands CNC (Conferación Nacional Campesina), in dem alle Ejidatarios zusammengeschlossen sind, wurden die Ejidobauern zur politischen Klientel und tragenden Säule des PRI-Systems.

Das Ejidoland besteht grundsätzlich aus zwei ungleichen Teilen: der größere Teil ist die Allmende und der kleinere Teil sind die Einzelparzellen. Jedes Ejidomitglied hat das individuelle Nutzrecht an seiner Parzelle, d.h. er besitzt eine ejidalen Landtitel, der ihn als Ejidatario ausweist und bebaut sein Land $\mathrm{zu}$ seinem eigenen Nutzen. Jede Parzelle und jeder Besitzer wird in einem speziellen, ejidalen Landbesitzkataster geführt (Registro Agrario Nacional). Die Allmende besteht in der Regel aus Weideland und/oder Wald. Sie steht allen Ejidomitgliedern ${ }^{7}$ zur gemeinschaftlichen Nutzung offen. Die Ejidatarios wählen alle drei Jahre aus ihrer Mitte eine Ejidoleitung (comisariado ejidal), die für die Geschäftsführung zuständig ist. Es finden regelmäßig Ejidoversammlungen statt, auf denen allgemeine Belange besprochen werden.

\section{Der Weltmarktkontext und die neue Weltagrarordnung (1970er-90er Jahre)}

Die Nachkriegsära lässt sich in zwei große Phasen unterteilen. Im Zeitraum 1940-70 überwog der Protektionismus, mit dem jedes Land seinen Nahrungsmittelmarkt schützte. Ein Welthandel mit Nahrungsmitteln war noch weitgehend unbekannt. ${ }^{8}$ Der Welthandel der Nicht-Industriegüter bestand vor allem aus agrarischen Rohstoffen (z.B. Baumwolle, Kakao, Kaffee).

\section{Entstehung des Welthandels mit Nahrungsmitteln}

Anfang der 1970er Jahre begann sich die Situation grundlegend zu verändern. ${ }^{9}$ Ab 1972 begann die Sowjetunion stetig steigende Volumen von Getreide aus den USA zu importieren. ${ }^{10}$ Als Folge der steigenden Nachfrage nach Getreide sowie des ersten Ölschocks von 1973 stiegen die Weltmarktpreise für agrarische Rohstoffe und Nahrungsmittel bis Ende des Jahrzehnts kontinuierlich an. Die dadurch gestiegenen Gewinnmargen auf dem sich ent-

7 Ebenso den Landlosen, die in der Ejidoversammlung einen Antrag auf Landnutzung stellen. Wenn die Versammlung mehrheitlich zustimmt, bekommt der Landlose den Status den comunero und darf auf einen zugewiesenen Teil der Allmende bebauen.

8 Auch wenn es Getreideexporte der USA in die vom Krieg zerstörten Landwirtschaften Europas gab. Diese hatten jedoch eher den Charakter von Entwicklungshilfe.

9 Diese Phase wird von Harriet Friedmann/Philip McMichael (1989: 103) als „second food regime" bezeichnet.

10 Diese Importe waren in Folge der Produktionsumstrukturierung der sowjetischen Landwirtschaft hin zu verstärktem Futtermittelanbau und Viehzucht zur Deckung der Nahrungsmittelversorgung der Bevölkerung notwendig geworden. 
wickelnden Weltmarkt für Nahrungsmittel lockten weitere Anbieter auf den Plan. In den USA wurden 24 Millionen Hektar Brachland unter den Pflug genommen, so dass die Erträge bis Ende der 70er Jahre um 25\% anstiegen. Ab 1976 gab die Europäische Gemeinschaft (EG) ihr Selbstversorgungskonzept auf und trat als großer Weizenexporteur in den Wettbewerb ein. In den 70er Jahren begann eine weitere, folgenschwere Entwicklung, nämlich die Änderung der Konsumgewohnheiten der Verbraucher hin zu einer verstärkt auf tierischen Eiweißen beruhenden Ernährung. In der gleichen Periode kamen nicht-traditionelle Agrarprodukte erstmals auf den Weltmarkt: Obst und Gemüse (frisch und gefroren) sowie Blumen.

In den 1980er Jahren konsolidierte sich diese neue internationale Agrarordnung. Neu hinzu kam eine Revolution der Anbaumethoden und -technologien bedingt durch die Entwicklung von Bio- und Gentechnologie. Dies führte im Ergebnis zu spektakulären Produktivitätsfortschritten, die nun zum entscheidenden Arsenal im weltweiten Kampf um neue Absatzmärkte zwischen den Großanbietern wurde. Der Eintritt der industrialisierten Länder auf den Weltagrarmarkt und die Herausbildung ihrer hegemonialen Stellung hat auch zu einer neuen internationalen Arbeitsteilung geführt. Während in der Nachkriegsordnung die industrialisierten Länder mehr auf Produkte der gemäßigten Klimazonen beschränkt waren, haben sie ihre Produktion nun diversifiziert. Die Entwicklungsländer hingegen haben die traditionellen Agrarrohstoffe durch rentablere Agrarprodukte wie Obst, Gemüse und Blumen ersetzt.

Die Phase der hohen Agrarpreise fand 1982 ein jähes Ende als ein Jahrzehnt des Preisverfalls begann. Auf den sich verschärfenden Wettbewerb reagierten die großen Agrarexporteure (USA, EG) mit steigenden Subventionen für die heimischen Agrarproduzenten, was einer Exportsubvention gleichkam: dank staatlicher Subventionen konnte z.B. die EG mit konkurrenzlos billigen Agrarprodukten neue Absatzmärkte gewinnen. Die ökonomische Rationalität orientierte sich auf den Versuch, den Verlust der Gewinnmargen aufgrund des Preisverfalls durch Mengenüberschüsse überzukompensieren. Das Prinzip der Garantiepreise förderte die Überschussproduktion. Der so geführte ruinöse Niedrigpreiskrieg auf dem Weltmarkt der Agrargüter führte zu erheblichen Überkapazitäten für einzelne Produkte (z.B. Getreide). 1992 fielen die Agrarpreise auf das niedrigste Niveau des Jahrhunderts. In diesem Kontext kommt nach sieben Jahren Verhandlung die Uruguay-Runde des GATT mit dem BlairHouse-Abkommen von 1993 zum Abschluss. Zum ersten Mal werden Agrargüter (ebenso wie Dienstleistungen) in die Verhandlungen mitaufgenommen.

\section{Folgen der neuen Weltagrarordnung für Lateinamerika und Mexiko}

In Lateinamerika lassen sich vier Ländergruppen unterscheiden, die je unter- 
schiedlich auf die exogenen Veränderungen reagierten und somit eine je spezifische Variante der Weltmarkintegration erlebten.

Die erste Gruppe besteht aus Ländern, die einerseits ihre Selbstversorgung mit Grundnahrungsmitteln aufrechterhalten konnten und zusätzlich auf dem Weltmarkt als Exporteure von Getreide und Obst oder Gemüse auftreten. Dazu gehören Argentinien und Uruguay. Die zweite Gruppe besteht aus Ländern, die ihre Nahrungsmittelsouveränität beibehalten und zusätzlich Exporteure von „neuen“ Produkten, hauptsächlich Obst und Blumen werden konnten, so Chile, Kolumbien, Ecuador, Paraguay. Die dritte Gruppe besteht aus Ländern, die sich zwar in die neue internationale Arbeitsteilung als Exporteure der "neuen“ Agrarprodukte integriert haben, aber gleichzeitig die Nahrungsmittelsouveränität für Grundnahrungsmittel verloren haben. Dazu gehören Mexiko, Brasilien, Costa Rica, Honduras, Guatemala. Eine vierte, große Gruppe lateinamerikanischer Länder exportiert weiterhin die traditionellen Agrarrohstoffe und hat sich nicht in die neue Agrarordnung integriert, wie El Salvador, die Dominikanische Republik, Haiti, Nicaragua, Peru, Bolivien, Panama. Diese Länder gehören zu den Marginalisierten der neuen Weltagrarordnung. Einige von ihnen haben zusätzlich das Problem, dass ihre Selbstversorgung mit Grundnahrungsmitteln nicht gesichert ist (Peru, Bolivien, Dominikanische Republik, Haiti, El Salvador), während andere (Bolivien, Panama) eine einigermaßen ausgeglichene Selbstversorgung gewährleisten konnten. In Gestalt dieser Ländergruppe kommt der ausschließende und selektive Charakter der Transformation der Weltmarktordnung zum Ausdruck.

\section{Merkmale des exportorientierten Agrarmodells}

Die neue Weltagrarordnung, die sich ab den 1970er Jahren entwickelte, hat in Lateinamerika zur kapitalistischen Umstrukturierung in der Landwirtschaft geführt. Ab Anfang der 1970er Jahre verlor die Landwirtschaft ihre Rolle als Lokomotive der lateinamerikanischen Volkswirtschaften und erlitt einen bleibenden Bedeutungsverlust. ${ }^{11}$ Die Mehrheit der Länder begann nun gezielt mit der Förderung des Exportsektors der Landwirtschaft. Die neue Exportorientierung führte zur Veränderung der Produktionsstruktur: traditionelle Agrarrohstoffe (Zucker, Baumwolle, Kaffee, Kakao, u.ä) wurden abgelöst von den neuen, rentableren Exportprodukten wie Obst, Gemüse, Blumen. Die neuen Agrarexportprodukte sind sehr kapitalintensiv, was ausländische Direktinvestitionen erforderlich machte. Die hochgradige Exportorientierung (im Falle Mexikos nach den USA) erforderte umfangreiche Anpassungen an den jeweiligen Verbrauchermarkt. Logistik (Standortauswahl), Pflanzzeiten, Verpackung, Transport, Technologie (Biotechnologie, Biogenetik, Agrartechnologie) und

111970 machten die Agrarexporte Lateinamerikas noch 43,7\% der Gesamtexporte aus; 1991 waren es nur noch $24,5 \%$. 
Marketing werden für die just-in-time-Produktion zurechtgeschneidert. Ohne eine transnationale Produktionsorganisation ist dies weder finanziell (wegen der hohen Investitionskosten) noch informationstechnisch und personell (nur fest im jeweiligen Abnehmerland implantierte Unternehmen sind im Besitz der notwendigen Marktinformationen und Kontakte) vorstellbar.

$\mathrm{Ab}$ Anfang der 1980er Jahre führte die neoliberale Politik allgemein zum Rückzug des Staates aus der Wirtschaft. Im Kontext der IWF-Strukturanpassungsprogramme ging es im Kern stets darum die Landwirtschaft dem „freien Spiel“ der Marktkräfte zu überlassen. In der Folge fielen die Produzentenpreise und gleichzeitig stiegen alle Inputkosten der landwirtschaftlichen Produktion. Die Landwirtschaft wurde unrentabel. Die neuen Markterfordernisse machten auch institutionelle Anpassungen notwendig. Der Wunsch, ausländisches Investitionskapital anzulocken und diesem eine ausreichende Rentabilität der Investition sichern zu können, ließen die Liberalisierung des Landund Arbeitsmarkts notwendig erscheinen. Schon ab den 1970er Jahren wurden auf dem Subkontinent Agrarreformen ,zurückgedreht” und damit den Kleinbauern (campesinos) ihre Grundlage entzogen. Dies führte zum Anstieg der Masse der Landlosen, deren Kämpfe um ein Stück Land seither überall zu mehr oder weniger gewaltsamen Konflikten geführt haben. ${ }^{12}$ In Mexiko kam die Agrarreform (1992) durch die Reform des Verfassungsartikels 27 auch de jure zum Ende. Der Weg zu einem neuen Typ der Landkonzentration, dem neolatifundio financiero, wird durch die Privatisierung des Ejido geebnet.

Der Bedeutungsverlust der Landwirtschaft als strategischer Wirtschaftszweig für die Entwicklung des Landes verlief in Mexiko besonders dramatisch ${ }^{13}$. Ab den 80er Jahren wurde es zunehmend schwieriger, ausländisches Kapital zur Investition in die Landwirtschaft zu bewegen. Das ausländische Anlagekapital, das wie eine Flutwelle über Mexiko und Brasilien hereinbrach, war größtenteils kurzfristiges Spekulationskapital, das den produktiven Sektor mied. Dem Exportboom bei den neuen, rentableren Agrarprodukten standen auf der anderen Seite steigende Importe von Grundnahrungsmitteln gegenüber. ${ }^{14}$ Auch

12 Chile unter Pinochet machte in dieser Entwicklung den Anfang. In Brasilien und Costa Rica kam es zu bedeutsamen Landkonzentrationen und die Zahl der Kleinbauern sank drastisch. In Nicaragua sorgte die Regierung von Violeta Chamorro (1990) dafür, dass die unter sandinistischer Herrschaft enteigneten Latifundien zum großen Teil wieder an die ehemaligen Besitzer gingen. Die Regierungen von Honduras (1992) und Ecuador (1994) verkündeten das Ende der Agrarreform.

131970 hatten die Agrarexporte einen Anteil von 37,49\% an den Gesamtexporten; 1990 waren es nur noch 9,88\%. Die Ablösung der traditionellen Agrarrohstoffe für den Export (Kaffee, Baumwolle, Zucker) durch die lukrativeren, neuen Agrarexportprodukte (Obst, Gemüse, Rinder) verlief rasant. 1980 lagen Mexikos Obst- und Gemüseexporte noch unterhalb der statistischen Erfassungsgrenze. 1985 begann die statistische Erfassung. 1990 war Mexiko auf Platz 4 der Weltrangliste für Tomatenexporte, auf Platz 8 für Trauben, auf Platz 6 für Frisch- und Gefriergemüse, auf Platz 11 bei Zitronen und Grapefruit.

14 Mexiko steht an 4. Stelle der Maisimporteure, an 2. Stelle der Sorgoimporteure. Auch bei 
der Rückzug des Staates aus dem Wirtschaftsgeschehen war in Mexiko radikaler als anderswo. Innerhalb von 8 Jahren (1986-94) werden die Verhältnisse in der Landwirtschaft auf den Kopf gestellt: Abschaffung der Garantiepreise (mit Ausnahme von Mais und Bohnen), Privatisierung von Staatsunternehmen in der Produktion (Zuckermühlen, Kaffee, Bewässerung), Kreditwesen und Versicherung (Banrural, ANAGSA), Vermarktung (CONASUPO), u.v.m. Hinzu kommt die abrupte Öffnung der Grenzen, die die einheimischen Agrarproduzenten mit der Konkurrenz der Billigimporte aus den USA konfrontierte. Das Modernisierungsprogramm für die Landwirtschaft (Programa de modernización para el campo, 1990-94) legte als neue Priorität die Förderung des Exportsektors fest und überläßt den Rest sich selbst. Die institutionelle Anpassung der Besitzverhältnisse in der Landwirtschaft an die Markterfordernisse nahm Gestalt an in der Reform des Artikel 27 der mexikanischen Verfassung. Der Schlußstein der neoliberalen Architektur war die Unterzeichnung des nordamerikanischen Freihandelvertrags TLC. Die in dem supranationalen Vertragswerk eingegangenen Verpflichtungen sollten den erreichten Stand der neoliberalen Liberalisierung irreversibel machen und somit den ausländischen Investoren mehr Sicherheit bieten.

\section{Das Privatisierungsprogramm PROCEDE (Programa de Certi- ficación de Derechos Ejidales y Titulación de Solares Urbanos)}

Das Jahr 1992 markierte die Wende. Das Ejido, die „heilige Kuh” der Mexikaner, wurde geschlachtet. Mit der Reform des Artikel 27 der mexikanischen Verfassung wurden die Voraussetzungen für die Privatisierung des Ejido geschaffen. Zur Durchführung des Privatisierungsprogramms PROCEDE wurden eigens zwei neue staatliche Institutionen gegründet: das Agrargericht (Tribunal Agrario) und die Agrarbehörde (Procuraduría Agraria). Das Agrargericht ist zuständig für die Schlichtung der zu erwartenden Landkonflikte und Grenzstreitigkeiten, während der Agrarbehörde die Durchführung von PROCEDE obliegt.

Laut Gesetz sollte die Durchführung von PROCEDE konkret so ablaufen: Vertreter der Procuradía Agraria stellen das Programm in der Ejidoversammlung vor, erklären die Funktionsweise und die einzelnen Schritte der Abwicklung. Die Mitglieder des Ejido debattieren die Vor- und Nachteile und stimmen schließlich darüber ab, ob das Ejido als Ganzes das Programm durchlaufen will. Nur mit Stimmenmehrheit der Versammlung kann PROCEDE in Gang kommen. Der erste Schritt ist die Landvermessung und Erstellung von Plänen der Parzellen sowie eine Liste der rechtmäßigen Ejidatarios mit zugehörigen Landbesitz ${ }^{15}$ (genaue Verortung der Parzelle, Grundfläche, welche

15 Hierbei sollen auch frühere Dokumente der Landvermessung (Karten, Estudio de usufructo parcelario) und Listen der rechtmäßigen Ejidatarios (Abschriften der Archive des Registro 
Nachbarn in jeder Himmelsrichtung). Diese kartographische und topographische Bestandsaufnahme wird vom staatlichen Institut für Statistik, Geographie und Informatik (INEGI) durchgeführt. Sobald diese Vorbedingung erfüllt ist, also genau bekannt ist, welcher Ejidatario wieviel Land besitzt und vor allen Dingen klar ist, wieviel Allmende das Ejido besitzt, kann zum nächsten Schritt übergegangen werden: Die gleichmäßige Aufteilung der Allmende unter den Ejidatarios. ${ }^{16}$ Laut Gesetz steht jedem Ejidatario zusätzlich zu seiner eigenen Parzelle der prozentuale Anteil an der Allmende zu. ${ }^{17} \mathrm{Ab}$ dem Augenblick, wo jeder Ejidatario seinen neuen Landtitel von PROCEDE ausgehändigt bekommt, ist er quasi-privater Besitzer seines Landes und kann damit machen was er will. Er darf nach Belieben verkaufen, verpachten, Hypotheken aufnehmen, joint-ventures mit Agrarunternehmen eingehen oder weiteres Land dazu kaufen. Auf diese Art und Weise dürfen sich auch Fremde im Ejido einkaufen, eine Sache, die früher nicht möglich war. Ehedem konnten im großen und ganzen nur erwachsene Kinder der alten Ejidatarios selbst Ejidatarios werden.

\section{Das neue Ejido}

Welche Folgen hatte nun die Reform des Artikel 27 und die neue Agrargesetzgebung von 1992 für das Ejido? Welches sind die wesentlichen Unterschiede zwischen dem alten und dem neuen Recht? Zum besseren Verständnis des „neuen“ Ejido nach 1992 sollen die Kernpunkte des alten und neuen Agrargesetzes gegenübergestellt werden, denn der Teufel steckt bekanntlich im Detail, insbesondere bei juristischen Sachverhalten.

\section{Fragmentierung des Landtitels}

Gemäß der alten Gesetzeslage erhielt ein Ejidatario einen ejidalen Landtitel für seinen Nutzrechtanteil am ejidalen Gesamtbesitz. Dieser Landtitel setzte sich aus zwei untrennbaren Bestandteilen zusammen: dem Recht auf die individuelle Parzelle (derecho sobre la parcela) und dem Recht auf die Mitbenutzung der Allmende (derecho sobre terrenos de uso común). Beide Bestandtei-

Agrario Nacional, R.A.N.) als Basis dienen.

16 Die Ejidoversammlung kann sich auch gegen die Parzellierung und individuelle Zuteilung von Allmendeland aussprechen und diese in Gemeinschaftsbesitz belassen. In der Praxis setzt sich aber meist die erste Variante durch und die Allmende wird aufgeteilt. Die Vertreter der Procuraduría Agraria werben auch geschickt für die Durchführung von PROCEDE, indem sie den Ejidatarios ein „Landgeschenk” in Form der aufgeteilten Allmende in Aussicht stellen.

17 Wenn zum Beispiel die durchschnittliche Parzellenfläche 8 ha beträgt, das Ejido aus 100 Ejidatarios besteht und die Allmende 1000 ha umfaßt, dann steht jedem Ejidatario zusätzlich zu seinen 8 ha Parzelle noch 10 ha anteilige Allmende zu. Die beiden neuen, ejidalen Landtitel (Derecho sobre la parcela und Derecho sobre tierra de uso común) von PROCEDE lauten demzufolge insgesamt über 18 ha Landbesitz. 
le des individuell erteilten Landtitels waren vollkommen gleichberechtigt, absolut untrennbar miteinander verbunden und konnten daher nur gemeinsam auf dem einzigen legalen Weg übertragen werden, dem der Erbschaft. Das neue Agrargesetz trennt nun die beiden Bestandteile. Es gibt zwei unterschiedliche Besitztitel, die je getrennt voneinander übertragen werden können. Der Ejidatario wird zum individuellen Besitzer an seiner Parzelle, die nun in eine neue juristische Kategorie des quasi-Privatrechts fällt. Damit wird die Parzelle zur beliebig handelbaren Ware auf dem Immobilienmarkt. Gleichzeitig erhält der Ejidatario ein Dokument, das ihn als Miteigentümer eines Gemeinschaftsbesitzes, der Allmende, ausweist, das ebenfalls individuell übertragbar ist. Der Besitzer irgendeines der beiden Titel wird zum anerkannten Ejidatario..$^{18}$ Diese Fragmentierung schafft die Möglichkeit, die Anzahl der Ejidatarios pro Ejido bei unveränderter Flächenausdehnung bis maximal auf das Doppelte zu erhöhen. Der Akkumulation von mehreren Rechtstiteln sind sehr lockere Grenzen gesetzt. Dies fördert die Konzentration von Land und damit von Macht in den Händen Einzelner. In welchem Ausmaß es zum An- und Verkauf von Ejidoland kommt, hängt wiederum von den Kosten alternativer Landkontrollmöglichkeiten (Pacht, Joint-venture) ab. Im neuen Agrargesetz bleibt die juristische Einheit und formale Identität des Ejido zwar gewahrt, aber seine soziale und ökonomische Grundlage verschwindet. Das Ejido hört auf ein Ganzes zu sein und zerfällt in die Summe seiner Teile. Wie jeder Umbruch von solch historischen Dimensionen hat dies Folgen für die Macht- und Interessenkonstellation vor Ort, also für die lokale (regionale) Politik. Wer kauft sich ins Ejido ein und mit welchem Ziel? Wie entwickelt sich das Verhältnis zwischen neuen und alten Ejidatarios? Führen die neuen legalen Möglichkeiten zum Machtverlust des Ejidovorstands (comisariado ejidal) oder zu seinem Gegenteil? Gewinnt oder verliert das Ejido als politische Institution an Gewicht im lokalen Machtgefüge?

\section{Besitzrecht an der Allmende}

Die wesentlichen Eigenschaften des Allmendelandes sollen auch nach der Verfassungsreform unangetastet bleiben. Der Gesetzestext steckt aber voller Widersprüche, so dass der eingangs deklarierte Anspruch an anderer Stelle wieder aufgehoben wird.

18 So Carlos Pérez Castañeda (1995: 486). In Sayula geschah etwas anderes: Besitzer von Allmendetiteln (derecho sobre terreno de uso común ) sind keine vollwertigen Ejidatarios, sie wurden aus dem Registro Agrario Nacional (R.A.N.) gestrichen und haben kein Stimmrecht in der Ejidoversammlung, sie haben den Status der comuneros. Nur die Besitzer von Parzellen-Titeln sind vollwertige Ejidatarios mit allen Rechten. Dies ist ein Beleg für die Widersprüchlichkeit der Umsetzung der Agrarpolitik. 
Unverjährbarkeit des Rechtsanspruchs (Imprescriptibilidad): Einerseits sichert das neue Agrargesetz die Unverjährbarkeit des Rechtsanspruchs für Allmendeland zu. Andererseits nimmt es diese Zusicherung wieder zurück, indem die Möglichkeit eingeräumt wird jenes Land (mit Ausnahme von Wald und Regenwald) durch gewohnheitsmäßige Nutzung zu erwerben. Die friedliche, ununterbrochene und öffentliche Inbesitznahme, sei sie in gutem Glauben oder wider Treu und Glauben erfolgt, produziert juristische Effekte, die schließlich in ein Eigentumsrecht münden. Dies bedeutet, dass man einen Besitzanspruch auf Allmendeland durch Verjährung bzw. durch Gewohnheitsrecht verlieren bzw. erwerben kann. Juristisch problematisch ist hier nur noch die Interpretation der Fristen von Verjährung.

Zur Verdeutlichung dessen, was die faktische Aufhebung der Verjährbarkeit des Rechtsanspruchs auf Allmende bedeuten kann, stellen wir uns einen konkreten Fall vor (der nicht frei erfunden ist). Beim Ableben eines Ejidatario geht der Rechtsanspruch auf den Landtitel häufig an seine Witwe über. Nehmen wir an, die erwachsenen Söhne der Witwe seien alle in die USA emigriert. Die Frau ist mit den jüngeren Kindern auf sich alleine gestellt, kann aber das Land nicht selbst bearbeiten und hat kein Geld, um jemanden dafür zu bezahlen. So bleibt das Land nach Ableben des Mannes zunächst unbestellt. Der expansionswillige Nachbar weiß um die Lage der Witwe, nutzt die Situation für sich aus, bestellt das Land in der nächsten Saison einfach mit und fährt die Ernte zu sich nach Hause. Nach einigen Jahren der zwar widerrechtlichen Inbesitznahme, aber ununterbrochenen Bearbeitung gehört das Land der Witwe ihm. Ihr Rechtsanspruch verjährt, wenn es ihr nicht gelingt, ihn vor Gericht durchzusetzen. Eine Durchsetzung dieser Art kostet Geld, das die arme Witwe nicht hat.

Unveräußerbarkeit von Allmende (inenajenabilidad): Das neue Agrarrecht garantiert auch weiterhin die Unveräußerbarkeit von Allmende, sie darf also nicht verkauft werden. Das Gesetz räumt der Ejidoversammlung jedoch das Recht ein, die Allmende zu parzellieren und die Fraktionen individuell oder gruppenweise den Ejidatarios zuzuteilen. Diese Zuteilung kann mit oder ohne Gegenleistung erfolgen. Eine finanzielle Gegenleistung im Austausch gegen die Zuteilung eines Anteils an der Allmende ist natürlich nichts anderes als ein Kaufakt. Die formal zugesicherte Unveräußerbarkeit der Allmende wird damit außer Kraft gesetzt.

Verbot der Pfändung (inembargabilidad): Das neue Agrargesetz erklärt, dass die Allmende nicht Gegenstand einer Pfändung sein kann. Das Nutzungsrecht an Teilen der Allmende darf aber an Unternehmen übertragen werden, so dass das Land Teil des Vermögens einer juristischen Person und somit zum Haftungskapital jener wird im Falle von Zahlungsunfähigkeit des Unternehmens. Andererseits erlaubt das neue Agrargesetz jede beliebige Form der 
Übertragung des Nutzungsrechts wie Pacht, Aufnahme einer Hypothek, Bürgschaft. Im Falle der Nichterfüllung der Vertragsbedingungen hat der Gläubiger das Recht den Garantiefall geltend zu machen und dies bedeutet die zeitweilige Pfändung des Nutzungsrechts. Auch wenn dies juristisch nicht exakt dieselben Konsequenzen hat wie den Verlust eines Besitzrechts, so sind die realen Effekte doch identisch. Dies gilt um so mehr, wenn es sich um die Einlösung hoher Verpflichtungen handelt, die einen langfristigen Verlust des Nutzungsrechts (20 und mehr Jahre) bewirken.

Unübertragbarkeit (intransmisibilidad): Das alte Agrargesetz garantierte die Unübertragbarkeit der ejidalen Rechte an der Allmende, indem es den Transfer von Besitz- oder Nutzrecht verbot. Das neue Agrargesetz erlaubt den Transfer von Besitz- und Nutzrechten in zwei Varianten: a) durch Parzellierung und individuell oder gruppenweise Zuteilung von Allmendeland. Dies wiederum ist Voraussetzung für den späteren Erwerb eines privatrechtlichen Landtitel; b) als Beitrag zum Kapital von Handelsgesellschaften (GmbH). Das Gesetz sichert den Ejidatarios im Konkursfall des Unternehmens das Vorkaufsrecht für das von ihnen eingebrachte Land zu. Dies bedeutet im Umkehrschluss, dass Ejidoland aus der Konkursmasse eines Unternehmens auch von Nicht-Ejidatarios käuflich erworben werden kann. Dies heißt aber, dass das fragliche Land vom Ejidoregime in das Regime des Privatrechts übergeht, ohne dass die für diese Konversion vorgeschriebene Prozedur eingehalten werden muß.

Unteilbarkeit (indivisibilidad): Im alten Agrarrecht war die Allmende unteilbar, sie durfte weder ganz noch teilweise parzelliert werden. Sie war der Kernbestand des Ejidovermögens, der immer wieder die Integration von Landlosen in den Kreis der Ejidatarios ermöglicht hatte. Ein landloser Bauer, der in der Gemeinde ansässig und guten Leumund bei den Ejidatarios genoß, konnte bei der Ejidoversammlung die Erlaubnis zum Urbarmachen und Bebauen eines Stück Lands auf der Allmende erhalten. Er erhielt damit den Status des comunero. Nach Ablauf von einigen Jahren und wiederum mit Zustimmung der Ejidoversammlung wurde jener comunero als Ejidatario mit vollen Rechten (Stimmrecht, Landtitel) im Ejido aufgenommen. Mit der Aufteilung der Allmende unter den nach 1992 zu einem bestimmten Zeitpunkt vorhandenen Ejidatarios wird der Zugang von Landlosen für die Zukunft verbaut.

\section{Das Besitzrecht an den Ejidoparzellen}

Die Veränderungen im neuen Agrargesetz haben für die Ejidoparzellen grundsätzlich dieselben Folgen wie für die Allmende. Hinzu kommt, dass von nun an der Ejidatario alleine (lediglich mit der Unterschrift von zwei Zeugen) über den eventuellen Verkauf oder Verpachtung an eine beliebige Person entscheiden kann, wobei Familienangehörigen nur ein Vorkaufsrecht eingeräumt wird. Ver- 
gegenwärtigt man sich die typische mexikanische Familie auf dem Land mit ihrer patriarchalen und autoritären Struktur, dann wird klar, dass die realen Möglichkeiten der Ausübung des Vorkaufsrechts durch Ehefrau oder Kinder des Ejidatario verschwindend gering sind. Wie sollte eine in der Regel nicht finanziell unabhängige Ehefrau eines Ejidatario die Mittel für einen solchen Kauf und den Mut für eine solche „Palastrevolution“ gegen ihren Ehemann aufbringen? Es bestehen jedoch größere Chancen, dass die Ejidoparzelle dem akuten Liquiditätsbedarf eines trunksüchtigen Ehemanns zum Opfer fällt und der Rest der Familie ohnmächtig dem Verlust ihrer Lebensgrundlage zusehen muß. Damit verliert die Ejidoparzelle den Charakter des Familienstammbesitzes (patrimonio familiar). ${ }^{19}$ Zusammenfassend lässt sich festhalten, dass das ejidale Parzellenrecht übertragbar, pfändbar, verjährbar, veräußerbar und teilbar ist. Das neu entstandene ejidale Parzellenrecht ist also ein individuelles Besitzrecht mit privatem Charakter mit einigen leichten Einschränkungen. Der Parzellenbesitz läßt sich als ejidaler Privatbesitz bezeichnen (propiedad privada ejidal).

Folgen für das gesellschaftliche Naturverhältnis: Diese Veränderungen des Rechtsregimes haben weitreichende Folgen für das gesellschaftliche Naturverhältnis. Während unter dem alten Gesetz ein Rechtstitelinhaber sich dem Land unterwerfen mußte durch den gesetzlichen Zwang zur ununterbrochenen Bearbeitung, kann sich der heutige Ejidatario von dieser Verpflichtung befreien ohne seinen Rechtstitel zu gefährden. Der ejidale Landbesitzer wird damit zum rein nominalen Besitzer und Rentier. Er verliert damit die Kontrolle über sein Land. Die Entscheidungen über das Land werden von den Managern des Unternehmens oder den größten Aktionären gefällt. So betrachtet, fallen direkte Kontrolle des Landes und Besitzrecht am Land auseinander. Der physische Kontakt von Mensch zu Land geht verloren in dem Maße wie sich das Geld zwischen beide schiebt. Der Besitztitel wird zum Anspruchstitel auf Anteil am Gewinn. Der Ejidatario wandelt sich vom stakeholder zum shareholder. ${ }^{20}$ Verschaffte das Land seinem Besitzer früher Nahrungsmittel, so bringt es ihm heute Geld ein. Dies hat Auswirkungen auf die Wertschätzung und Behandlung von Land, den Stellenwert von Geld, Konsumgewohnheiten (es wird gegessen was billig ist, nicht das, was geerntet wird), soziale Beziehungen und vieles mehr.

19 Hiermit bricht das neue Agrargesetz mit einer juristischen Tradition, das den Familienbesitz als eigene, unter besonderem Schutz stehende Besitzkategorie anerkennt (vgl. auch: homestead im US-Recht von 1862 und 1895; bien de famille im französischen Recht von 1909). Der Familienbesitz sollte vor der Willkür einzelner Familienmitglieder (in der Praxis: den Männern) geschützt werden, zugunsten des Erhalts der Lebensgrundlage der Familie als Gesamtheit. Das reformierte Agrargesetz orientiert sich nun an den Normen des Zivilrechts, das dem Eigentümer einer Sache die volle Verfügungsgewalt über sie einräumt.

20 Eine Analyse dieses Wandels für den Fall der Industriebetriebe findet sich in Altvater/ Mahnkopf (1996, Kap.9). 
Folgen für die Sozialbindung von ejidalem Landbesitz: Im alten Agrarrecht konnte nicht jeder Beliebige Ejidatario werden. Der Zugang zum Ejido via Landzuteilung und Ejidoneugründung war an die Erfüllung von bestimmten Kriterien gebunden, die die Gruppe der möglicherweise Begünstigten sozial eingrenzte auf die Bauernschaft. Von daher ist das alte Agrarrecht zurecht als ein Klassenrecht, nämlich zugunsten der Bauern bezeichnet worden. Im neuen Agrarrecht kann fast jeder Beliebige Ejidatario werden. Hiermit wird das Tor zur Auflösung der Bauern als sozialer Klasse geöffnet und der Staat als Schutzpatron ihrer Existenz entfällt. Das ehemalige Klassenrecht verwandelt sich in ein Individualrecht. Im alten Agrarrecht waren die Rechte der Ejidatarios an eine Reihe von Verpflichtungen geknüpft, ${ }^{21}$ erlassen wurden im Hinblick auf das Schutzinteresse des Staates zugunsten von sozial Benachteiligten wie Witwen, Waisen, Behinderten und Arbeitsunfähigen. Damit wurde der soziale Charakter des ejidalen Besitzrechts unterstrichen. Wie schon deutlich geworden ist, wurden diese Verpflichtungen im neuen Agrarrecht ersatzlos gestrichen. Die ejidalen Landbesitzer wurden den privaten gleichgestellt. Damit verliert das Landeigentum seine soziale Funktion, die ihm von der Verfassung von 1917 zuerkannt wurde. Dies bedeutet, dass das gesellschaftliche Allgemeininteresse nicht mehr als Aufrechterhaltung der landwirtschaftlichen Produktion und Sicherung der nationalen Nahrungsmittelsouveränität definiert wird.

\section{Der Fall Sayula/Jalisco}

Am Fall des Ejido Sayula ${ }^{22} / J a l i s c o$ kann man beobachten: das reale Ergebnis der Abwicklung des Privatisierungprogramms PROCEDE hängt von lokalen Faktoren (Machtverhältnisse, Interessenlagen, ökologischen Rahmenbedingungen, aktuelle Wirschaftskonjunktur) ab. Die Privatisierung des Ejido Sayula verlief mit Nebeneffekten, die auf die Natur des PRI-Systems hinweisen: es gab Landraub, Betrug und Mord. Entgegen den Hoffnungen der einen und den Befürchtungen der anderen hat die Privatisierung von Ejidoland in Sayula nicht zu massiven Landverkäufen geführt, das Land wird hauptsächlich

21 Die Handlungspflichten umfassten 1) gegenüber der Familie: Unterhaltsverpflichtungen gegenüber der Ehefrau und den minderjährigen Kindern des Ejidatario oder im Falle seines Ablebens gegenüber der Witwe und den Waisen; 2) gegenüber dem Ejido: Verpflichtung zur Teilnahme an den Ejidoversammlungen, Verwendung von Kredit für beantragte Zwecke, Bebauung der Parzelle mit den programmatisch festgelegten Kulturen, Erledigung von Gemeinschaftsaufgaben, Vertrieb der Ernte über das Ejido; 3) gegenüber der Gesellschaft: Verpflichtungen zur ununterbrochenen Nutzung des Landes (Verbot der Brache). Die Unterlassungspflichten umfassten 1) gegenüber dem Ejido: Verbot des Verkaufs oder Verpachtung von Ejidoparzelle bzw. Allmendeanteils, Verbot der Beschäftigung von Lohnarbeitern; 2) gegenüber der Gesellschaft: Verbot des Anbaus von Rauschmitteln.

22 Sayula ist eine Kleinstadt von ca. 30.000 Einwohner, $100 \mathrm{~km}$ südlich von Guadalajara (4 Mio. Einwohner) im Staat Jalisco. Das Ejido Sayula hatte 1998 ca. 110 Mitglieder. 
verpachtet. Die Entscheidung für Pacht oder Nicht-Pacht wird nicht nach Marktkriterien gefällt, vielmehr spielt die gesunkene ökologische Qualität (Erosion, Wassermangel) die entscheidende Rolle. Als Pächter traten in den 1990er Jahren die Agromaquilas auf den Plan. Die Schuldenkrise der Großbauern und die Privatisierung von Ejidoland sind zusammen die Voraussetzungen für die Transnationalisierung der mexikanischen Landwirtschaft.

Die Probleme des Ejido Sayula begannen 1993 mit der Durchführung von PROCEDE. ${ }^{23}$ Die Regierung hatte für dieses Privatisierungsprogramm mit dem Versprechen von „mehr juristischer Sicherheit am Landbesitz“ geworben. Doch in Sayula kam alles anders, bei der Abwicklung des Privatisierungsprogramms kam es zu erheblichen Unregelmäßigkeiten:

- Geldunterschlagungen aus der Ejidokasse aus dem Holzverkauf von Ejidowald und aus Entschädigungszahlungen für vom Staat enteignetes Ejidoland, auf dem das in Sayula ansässige 14. Regiment des nationalen Heers seine Kaserne errichtet hat;

- von der aufgeteilten Allmende (fondo común) wurde manchen Ejidatarios überproportional viel Land gegeben, während andere leer ausgingen;

- Landraub: im Rahmen der Neuvermessung des Ejidolands - vorbereitender Schritt für die Umverteilung und Landtitelvergabe - wurden uralte Grenzen so verschoben, dass einigen Ejidatarios Land von ihrer Parzelle abgezweigt wurde. Die so entstandenen „neuen“ Parzellen wurden sofort an Dritte weiterverkauft. Von insgesamt 112 Ejidatarios sind 30 von solcher Art Landraub betroffen, davon haben 11 ein Gerichtsverfahren vor dem Agrargericht (Tribunal Agrario) angestrengt. Nach ca. 5 Jahre entschied die letzte Instanz zugunsten der Geschädigten;

- Streichung von alteingesessenen Ejidatarios aus der Liste des Registro Agrario Nacional (R.A.N.): einige der Ejidatarios, die schon seit mehr als 20 Jahren auf ihrer Parzelle sind, tauchten in der Liste des R.A.N. von 1996 plötzlich nicht mehr auf;

- willkürlich selektive Aushändigung der neuen PROCEDE-Landtitel: einige der alten Ejidatarios warteten 1998 noch immer auf die neuen Landtitel, während alle „neuen” Ejidatarios schon seit Mai/Juni 1995 im Besitz dieser Papiere waren. Ohne die neuen Landtitel können die Ejidatarios keine vom Staat gewährten Hilfen für die Landwirtschaft beantragen (PROCAMPO).

Vordergründige Akteure dieser Unregelmäßigkeiten waren die Mitglieder der ehemaligen Ejidoleitung (1992-95). Vom Landraub betroffene Ejidatarios haben Rat und Unterstützung bei der Procuraduría Agraria gesucht. Vergeblich. Von Anfang an kämpften die Betroffenen mit ihren schwachen Mitteln um ihre Landrechte: sie haben unzählige Bitt- und Protestschreiben bezüglich der Mißstände an die verschiedenen Verantwortlichen der Procuraduría Agraria,

231993 hatten in Jalisco 300 von insgesamt 1370 Ejidos das Programm PROCEDE durchlaufen. 
Ministerien, Gouverneur und andere Regierungsmitglieder von Jalisco und sogar an Präsident Zedillo gesandt, Presse und TV eingeschaltet, Anwälte bezahlt. Dies bedeutete einen enormen Aufwand an Zeit und Geld. Höhepunkt des Landkonflikts war die Ermordung des Sprechers der inconformes: ${ }^{24}$ Raúl González Vázquez wurde am Morgen des 24. Januar 2000 von zwei gedungenen Pistoleros vor seinem Haus erschossen. Die Untersuchung des Mordfalls wurde im November 2000 ergebnislos eingestellt.

Wichtig für das Verständnis der vorgefallenen Unregelmäßigkeiten, insbesondere des Landraubs, ist die Ejidoversammlung vom 11. Februar 1995. Diese Ejidoversammlung war eine sogenannte asamblea dura ${ }^{25}$ in der es um die „Grenzziehung, Bestimmung und Zuweisung von Ejidoland“ (delimitación, destino y asignación de las tierras ejidales) ging. In dieser Versammlung sollten die rechtmäßigen Ejidatarios als Besitzer ihrer Parzellen bestätigt werden und ihnen der prozentuale Anteil an der Allmende zugewiesen werden. Die neuen Landtitel von PROCEDE, die die Privatisierung des Ejidoland besiegeln, beruhen auf den Beschlüssen solcher asambleas duras. Angesichts der Bedeutung solcher Versammlungen schreibt der Gesetzgeber die Anwesenheit eines staatlich anerkannten Notars und anderer Behördenvertreter vor. $\mathrm{Zu}$ Beginn der Versammlung vom 11. Februar 1995 wurde wie üblich zunächst das Quorum festgestellt. Denn nur mit dem erforderlichen Quorum ist die Versammlung beschlußfähig. Genau hier setzte die betrügerische Manipulation der Ejidoleitung ein: die vorgelesene Anwesenheitsliste enthielt über 30 Personen, die auch physisch in der Versammlung anwesend waren, aber keine Ejidatarios waren. Die rechtmäßigen Ejidatarios bemerkten sofort diese Manipulation und protestierten. Das notariell beglaubigte Sitzungsprotokoll übergeht diese Problematik und den Protest der wahren Ejidatarios. Die Allmende wurde auf alle Anwesenden vom 11. Februar 1995 aufgeteilt - also auch auf Personen, die keine Ejidatarios waren und sind - und die vom INEGI falsch vermessenen Grundstücke wurden von der Versammlung dank des herbeigebrachten Stimmviehs mehrheitlich bestätigt.

In diesem Zusammenhang tut ein Hinweis not: am 12. Februar 1995, also genau einen Tag nach der Ejidoversammlung, fanden die Stadtratswahlen statt. Erstmals in der Geschichte Sayulas gewann ein Oppositionskandidat von der konservativen PAN. Am 11. Februar 1995 jedoch ergriff der PRIKandidat das Wort in der Ejidoversammlung und wandte sich an die Anwesenden mit den Worten: „Compañeros, wer heute hier anwesend ist und mitstimmt, der ist jetzt schon rechtmäßiger Ejidatario. Ich erwarte, dass Ihr mir morgen Eure Stimme gebt.“ Mit der Versammlung vom 11. Februar 1995

24 So die Selbstbezeichnung der vom Landraub betroffenen Ejidatarios.

25 Der „asamblea dura“ vom 11.2.1995 folgten weitere; am 30.11.1997 und 1.2.1998, in denen genau dasselbe passierte. 
wurde der Landraub im Mitwissen der Vertreter der Procuraduría Agraria und des wahrscheinlich bestochenen Notars offiziell urkundlich beglaubigt.

\section{Welche Bedeutung hat die lokale Ejido-Privatisierung via PROCEDE für die nationale Politik?}

Landkonflikte im Rahmen der Privatisierung des Ejido via PROCEDE sind systemisch angelegt. Dies hat historische Wurzeln (Jean Meyer 1973). Daran ändert der neoliberale Kontext nichts, im Gegenteil, er verschärft die Lage durch Verknappung von sämtlichen Ressourcen (ökonomisch, politisch, ökologisch, sozial) für lokale Mächtige, was seinerseits die Verteilungskonflikte schürt. Eine Selbstorganisation der PROCEDE-Geschädigten analog zur Schuldnerbewegung El Barzón als Selbstorganisation der Bank-Geschädigten ist ausgeblieben. Einer der Gründe dafür liegt bei den Ejidatarios selbst: sie sind ärmer, unwissender, ohnmächtiger als die wohlhabenderen Barzonistas. Ein anderer Grund hängt mit der Herrschaftsstruktur des politischen Systems zusammen: sie ist gespalten nach Klasse und Rasse. Ein Ende der impunidad (Straflosigkeit) für staatlich verantwortete oder staatlich induzierte, politische Gewalt und somit ein Schritt in Richtung eines demokratischen Rechtsstaats in Mexiko wäre im Kontext der Landfrage nur möglich, wenn konkurrierende politische Kräfte der PRI das Ejido als „domaine réservé“ streitig machten. Einer der wichtigsten Hebel wäre hierbei eine autonome Judikative als eine der drei Staatsgewalten.

\section{"Still the century of corporatism?"26 Für die einen ja, für die anderen nein.}

Mit der Durchsetzung der neoliberalen Politikreformen in der Landwirtschaft nahm das autoritäre PRI-Regime die „politischen Kosten“ eines solchen Wandels billigend in Kauf. Das Regime selbst sperrte die ehemaligen Kommunikationskanäle der korporatistischen Verhandlungen zu. Am Beispiel von Sayula sehen wir die von Hubert C. de Grammont (1998) aufgestellte These von der Aufspaltung des Regimes und seiner Herrschaftspraxis entlang der Klassenlinien bestätigt. Für die Armen (immerhin mehr als 50\% der Bevölkerung) funktioniert weiterhin der Korporatismus. Die nationalstaatlichen Politiken zur Abfederung der „sozialen Härten“ der neoliberalen Politik äußern sich auf dem Land in Gestalt der Institutionen wie PRONASOL und PROCAMPO. Die Reform der institutionellen Rahmenbedingungen auf dem Land als Reaktion auf die Sachzwänge der Globalisierung nimmt Gestalt an in der Reform des Artikel 27 der mexikanischen Verfassung via PROCEDE. Für die Mittel- und Oberschicht (Reiche) auf dem Land und in der Stadt glei-

26 Der Originaltitel von Philippe C. Schmitter (1974: 85-131) ist als Aussage formuliert. 
chermaßen gilt ein anderes Regime: die alten korporatistischen Kontrollmechanismen verschwinden und die Parteiendemokratie (democracia electoral) mit sauberen Wahlen und Parteienkonkurrenz werden zum einzigen Weg der Interessenartikulation und institutionellen Möglichkeit die eigenen Forderungen gegenüber dem Staat durchzusetzen.

\section{procede, procampo, pronasol und der Korporatismus}

Die Funktionsweise der Institutionen PROCAMPO ${ }^{27}$ und PROCEDE ist eher klientelistisch, autoritär, korporatistisch, repressiv und funktioniert somit nach dem alten Beziehungsmuster des autoritären PRI-Regimes. Die Mechanismen der sozialen Kontrolle bleiben erhalten oder können sich sogar verstärken. In Sayula können wir im Fall von PRONASOL ${ }^{28}$ eine gegenläufige Bewegung zu dieser Tendenz beobachten. ${ }^{29}$ Die Abwicklung von PROCEDE zeigt am deutlichsten das Beharrungsvermögen der alten Kontrollmechanismen des PRI-Regimes, die sich sogar auf dramatische Weise verschärften. Für die Abwicklung des Privatisierungsprogramms im Ejido sind die traditionellen Akteure der Landwirtschaftspolitik (Agrarministerium SAGAR, INEGI, RAN) und die neu geschaffenen Institutionen (Procuraduría agraria, Tribunal agrario) auf der nationalen bzw. regionalen Ebene, sowie die Ejidoleitung auf der lokalen Ebene zuständig. Die personelle Kontinuität von den alten zu den neuen Institutionen ist gewährleistet (ehemaliges Personal der SAGAR und SARH wurde von der Procuraduría agraria übernommen), so dass die alten Beziehungsgeflechte intakt geblieben sind. Das Politikergebnis ist das geschilderte.

Definiert man den mexikanischen Korporatismus auf dem Land der postrevo-

27 PROCAMPO ist eine Art staatliche Beihilfe zum Lebensunterhalt für Landwirte. Laut Gesetz soll es an Agrarproduzenten ausgezahlt werden, in der Realität geht das Geld an die Landbesitzer, unabhängig davon, ob sie das Land selbst bebauen oder verpachten.

28 PRONASOL (Programa Nacional de Solidaridad) war die Hauptinitiative der Regierung Salinas (1988-94) im Bereich Sozialpolitik.

29 Ab dem Zeitpunkt, wo die „alternancia” (der politische Machtwechsel) im Rathaus erstmals geglückt war (ab 1995 mit der ersten PAN-Stadtregierung), kann das alte Beziehungsmuster aufgebrochen werden. An die Stelle der mit dem PRI-System identifizierten Akteure und Beziehungsnetze treten die neue Regierungsmannschaft im Rathaus und ihre „amigos“ in anderen wichtigen Hierarchien auf lokaler und regionaler Ebene. Die langjährige Forderung der Gesellschaft von Sayula nach Transparenz der Konten, Öffentlichkeit der Entscheidungsmechanismen, demokratischer Beteiligung der Betroffenen an PRONASOL-Projekten kann nun endlich durchgesetzt werden. Die demokratischen Prozeduren werden korrekt eingehalten, der neue PAN-Bürgermeister legt großen Wert auf Transparenz seiner Amtsführung und Beteiligung der Bevölkerung am Politikprozess in der Absicht, das verloren gegangene Vertrauen der Bevölkerung in die öffentlichen Institutionen wieder zurück zu gewinnen. Es geht explizit um die Wiederherstellung der verlorengegangenen Legitimität des lokalen politischen Systems. Für die neue PAN-Regierung wird dieser Aspekt ihrer alltäglichen Arbeit an der ,gobernabilidad" (Regierbarkeit) im Municipio zum Überlebenstraining. PRONASOL wird zum zentralen Beweisinstrument ihrer neu- und andersartigen Amtsführung und somit zum Baustein für die Legitimität des neuen Stadtregimes. 
lutionären Phase im Sinne von Hubert C. de Grammont (1998) als soziales System, das auf folgenden Basiseinheiten beruht: 1) Bauernfamilie, 2) Ejido (bzw. comunidad agraria) für den „sozialen Sektor ${ }^{\text {“30 }}$ und 3) Organisationen der privaten Landbesitzer (nationaler Dachverband; regionale Verbände; lokale Viehzüchter-, Schweinezüchter-, Landbesitzervereine), dann wird klar, warum dieser Korporatismus für den Fall der privaten Landwirte sich in Auflösung befindet bzw. mancherorts schon völlig verschwunden ist, während der „soziale Sektor“ eher unter einem härteren Zugriff zu leiden hat. In vergangenen Zeiten des unangefochtenen PRI-Monopols hat der Korporatismus auf dem Land gleichzeitig mehrere Funktionen erfüllt, nämlich die konkurrenzlose, da einzige Organisationsform für produktive (also landwirtschaftliche), soziale und politische Zwecke der Landbevölkerung. Die politische Beziehung des Staates zu den Agrarproduzenten (sowohl für Ejidatarios als auch für private Landwirte) war nicht auf dem Zusammenschluss freier Individuen in politischen Parteien gebaut, sondern beruhte auf dem Prinzip der Repräsentation von sozialen Klassen (hier: Bauern als Klasse) und dem vorausgesetzten Konsens unter ihnen. Daher wurden die Ejidatarios, ohne Ansehen der bestehenden Unterschiede unter ihnen, in einer einzigen nationalen Organisation (Confederación Nacional Campesina, CNC) zusammengefaßt. Auf Seiten der privaten Landbesitzer kam dasselbe Organisationsprinzip zur Anwendung. Über 50 Jahre spielte das Ejido (via CNC) und die Verbände der privaten Landbesitzer (CNPR) die Rolle der parastaatlichen Vermittlungsorgane, d.h. sie dienten als permanenter Kommunikationskanal zwischen dem Staat (vertreten durch das Agrarministerium) und der Bauernschaft. Der so institutionalisierte Korporatismus war aber von seinem Wesen her ein höchst komplexes Beziehungssystem, das verschiedene Elemente miteinander verband: „große“ Abkommen zwischen der Bauernschaft und dem Staat bezüglich ihrer Hauptforderungen (nach Land); Formen der legalen Beschränkung (Artikel 27) eben dieser Forderungen sofern sie andere Machtgruppen betrafen (Hacendados); offene Repression in Krisenmomenten, die alles zulässt von der Verhaftung über Mord bis hin zum Einsatz des Militärs. Der mexikanische Korporatismus (auf dem Land) bestand also aus den vier Elementen, die in wechselnder Gewichtung miteinander kombiniert wurden: 1) Verhandlung; 2) soziale Kontrolle; 3) soziale Repräsentation nach dem Konsensprinzip; 4) Repression. Dieser Korporatismus gerät in die Krise mit dem Auftauchen des neoliberalen Staates, er wird dysfunktional. Die Kommunikationskanäle werden „von oben“ dicht gemacht.

\section{Die Natur des politischen Systems zeigt sich am Landproblem. E-}

30 Wörtliche Übersetzung von „sector social“; gemeint ist der kollektive Landbesitz im Ejido bzw. der comunidades agrarias. 


\section{jidatarios und landlose Bauern}

Das Zusammenspiel der neoliberalen Reformen auf dem Land und die Effekte der Globalisierung in der Landwirtschaft haben auf der unteren Stufe der sozialen Hierarchie in Sayula zu unterschiedlichen Auswirkungen auf Ejidatarios und landlosen Bauern geführt. Angesichts des Ausmaßes der Wirtschaftskrise insbesondere ab 1995 werden Ejidatarios als klientelistische Adressaten dieser Politiken zu relativ Privilegierten im Vergleich zu den landlosen Bauern nach dem Motto „Unter Blinden ist der Einäugige König“. Der PROCEDE-Titel weist den Ejidatario als Landbesitzer aus und verschafft ihm Zugang zu PROCAMPO-Geldern und zum Landmarkt. Obwohl PROCAMPO laut Gesetz prinzipiell auch an Landlose ausgezahlt werden kann, wird es in Sayula ausschließlich an die Landbesitzer vergeben. Durch die Aufteilung und Parzellierung der Allmende des Ejidos unter den Ejidatarios via PROCEDE ist grundsätzlich die Neuaufnahme von landlosen Bauern im Ejido für alle Zukunft verbaut. Als Folge von PROCEDE entsteht ein Angebot von ejidalem Pachtland ${ }^{31}$, das von den Agrokonzernen genutzt wird. Die Form der Landkontrolle via Pacht kommt ihren Produktionsinteressen entgegen. Die Ejidatarios, die ihr Land verpachten, haben Vorrang bei der Einstellung als Tagelöhner. Auf diese Weise können sie im günstigsten Fall PROCAMPO, Pachtzins und Lohn kumulieren und eventuell auf einem verbliebenen Stück Land Grundnahrungsmittel für die Selbstversorgung anbauen. Die landlosen Tagelöhner müssen alleine mit dem Lohn ihrer Arbeit auskommen. Der sozioökonomische Effekt dieser neoliberalen Politiken ist - wie Eric Léonard (1998) es formuliert - das „Einfrieren der Sozialstruktur" unter der armen Landbevölkerung. PROCEDE und PROCAMPO wirken in Richtung auf eine Polarisierung der unterschiedlichen Akkumulationsmöglichkeiten unter der ärmeren Landbevölkerung, indem sich die Lebensbedingungen und Produktionspotentiale der Landlosen erheblich verschlechtern. Vereinzelte Chancen für den sozialen Aufstieg haben die Kinder dieser Landlosen über den Zugang zu höherer Bildung.

\section{Das Ejido als "domaine réservé" der PRI}

Ausgehend von der allgemeinen Prämisse, dass wer die ökonomischen Ressourcen kontrolliert auch die politische Macht hat, läßt sich aufdecken wie und warum der PRI-Korporatismus/Klientelismus auf dem Land geschwächt bzw. gestärkt wird. Die Kontrolle über PROCEDE und PROCAMPO hat der „alte Apparat" des PRI-Regimes (Procuraduría agraria, Tribunal agrario, Registro Agrario Nacional, INEGI) auf regionaler und nationaler Ebene und die Ejido-

31 Verpachtet werden hauptsächlich die schlechtesten Böden an die Agavenplantagen. Das liegt daran, dass die Entscheidung zwischen „Pacht“ oder „Nicht-Pacht“ nicht nach Marktkriterien getroffen wird. Verpachtung kommt nur in Frage, wenn das Land aufgrund ökologischer Schädigung keine Ernte mehr hervorbringt. 
leitung (comisariado ejidal) auf lokaler Ebene. Mittels der neoliberalen Politikreformen geht es also um die Rekonstruktion der traditionellen korporatistischen und klientelistischen Kontrollmechanismen rund um den ökonomischen Faktor „Landzugang“. Die mit der Durchführung der neoliberalen Reformen betrauten staatlichen Institutionen gehören samt und sonders der PRIDomäne an, langjährig eingespielte Beziehungsnetze reproduzieren die alte politische Kultur. Dass das PRI-System bei den Wahlen in die Defensive geraten ist und sich daher in einer Position der relativen Schwäche befindet, verschärft den korporatistischen Zugriff nur noch. Und der durch neoliberale Sparmaßnahmen verringerte Verteilungsspielraum erklärt, warum die Kontrolle der Klientel weniger durch „carrots“ (Belohnungen) als durch „sticks“ (Repression) ausgeübt wird. Dies geschieht jedoch auch aus profan ökonomischen Gründen: wo die Arbeitsplätze von PRI-Funktionären und Angestellten der Agrarbehörden gefährdet sind und die Kaufkraft ihrer Gehälter dahinschmilzt, sind sie um so empfänglicher für Bestechungsgelder, widerrechtliche Aneignung von Ejidoland und andere Gesetzesübertretungen. Der Fall Sayula zeigt, dass es hier zur Überschneidung von privaten, ökonomischen Interessen der PRI-Vertreter an einem Stück Land oder illegalem Zusatzeinkommen auf der einen Seite und dem politischen Überlebenswillen der PRI als Partei auf der anderen Seite gibt. ${ }^{32}$

\section{"Exit" oder "voice" oder beides}

Wie Jonathan Fox (1994: 243-276) richtig bemerkt, gibt es zwei allgemeine Trends: Es geht um die grundsätzliche Alternative von „exit“ und „voice“. In Sayula haben wir gesehen, dass die meisten der Armen auf dem Land mit einer kombinierten familiären Überlebensstrategie reagieren: ${ }^{33}$ Stellt man sich die Alternative zwischen „exit“ und „voice“ als ein „entweder - oder“ vor, so geht man gedanklich vom Individuum aus. In der mexikanischen Realität leben die Menschen aber nicht alleine, sondern in Familien und handeln dementsprechend kollektiv. Betrachtet man die Familie als Akteur, dann kommt „exit“ und „voice“ parallel und in Mischform vor. So sind z.B. die selbstorganisierten

32 So gibt es Hinweise darauf, dass die Gelder aus dem illegalen Verkauf des geraubten Ejidoland im Ejido Sayula zur Finanzierung des Wahlkampfes der PRI (1995) verwendet wurden. Angesichts leerer Parteikassen überließ die regionale/nationale Parteispitze es den lokalen Vertretern (CNC, Ejidoleitung) für eine finanzielle Ersatzquelle zu sorgen.

33 Sie beinhaltet Auswanderung in die großen Städte oder in die USA auf der Suche nach Lohnarbeit; illegale, aber gewinnbringende Tätigkeiten wie Rauschmittelanbau und Handel damit; illegaler Holzeinschlag und Handel damit; Prostitution; vermehrte Eigenarbeit (zurück zum Holzfeuer, weil Gas zu teuer ist; Handwäsche statt Maschinenwäsche; Selbstnähen statt Kleiderkaufen); Kumulieren der Lohneinkünfte durch Kinder- und Frauenarbeit; Änderung der Konsumgewohnheiten (Soya statt Fleisch; Wasser statt Softdrinks); selbstorganisierte Basisgesundheitsversorgung statt Artzbesuche; alleinerziehende Mütter statt „komplette“ Familien; selbstzerstörerische Aggression wie Alkoholismus und Drogenkonsum (v.a. Thinner-Schnüffeln bei Kindern und Jugendlichen); Jugendbandenkriminalität; intrafamiliäre Gewalt vor allem gegen Frauen; eigenmächtige Abtreibungen mit Todesfolge. 
Gruppen wie Basisgesundheit, Sparvereine, Frauengruppen, Hausbaugruppen potentiell politisch aktiv und daher sowohl „exit“ (aus der Landwirtschaft) als auch „voice“. In Sayula haben sich diese hauptsächlich von Frauen getragenen Vereine und sozialen Bewegungen regelmäßig in die Lokalpolitik eingemischt und in Zeiten der PRI-Herrschaft diese durch Denunzierung der Mißstände herausgefordert. ${ }^{34}$ Diese verheirateten Frauen (,las señoras") arbeiten - quasi per Definition, weil sie Frauen sind - nicht in der Landwirtschaft (weder bezahlt noch unbezahlt), ermöglichen aber durch die Reduzierung der Geldausgaben für die Haushaltsführung und durch die organisierte Selbsthilfe dem Ehemann weiterhin als selbstständiger Bauern (Ejidatario) oder Tagelöhner in der Landwirtschaft tätig zu bleiben. Durch Einsparen, „voice“-Aktivitäten und Einspringen bei Bedarf als zusätzliche Arbeitskraft (Ernte) tragen die Frauen dazu bei, dass die Männer in der Landwirtschaft bleiben können.

Wahr ist aber auch, dass es für die von PROCEDE betroffenen Ejidatarios (noch?) keine entsprechende „voice“-Aktivität gibt wie für die überschuldeten Großbauern in Gestalt des El Barzón. Dass die der Option „exit“ eindeutig Vorrang besitzt, läßt sich für den Fall Sayula jedoch nicht bestätigen. Betrachtet man den familiären Haushalt als Untersuchungseinheit und über längere Zeit (im Idealfall den gesamten Familienzyklus), so wird klar, dass die Bauern von der Landwirtschaft nicht loskommen, jedenfalls nicht für immer. Ein Stück Land im Familienbesitz ist ein wertvolles Gut, da es zumindest die Versorgung mit Grundnahrungsmitteln sichert. Die „exit“Option hieße konkret Lohnarbeit, doch davon gibt es erstens nicht genug für alle und zweitens werden Hungerlöhne gezahlt, von denen man keine Familie ernähren kann.

Dass die Probleme des Ejido in Sayula nicht zum Politikum wurden, hängt auch damit zusammen, dass es ein unausgesprochenes „gentlemen's agreement“ gibt: das Ejido gilt als „domaine réservéc der PRI, während die anderen Parteien sich um den Rest kümmern. Die ausgebliebene Politisierung des Landproletariats und der Ejidatarios für bäuerliche Ziele im engeren Sinne ist also eine Folge mehrerer Faktoren: 1) Tagelöhner der Agromaquilas sind hauptsächlich Jugendliche ohne gewerkschaftliche Erfahrung; hoher turn-over des Personals und saisonal beschränkte Arbeitszusammenhänge; sofortige Repression gegen Organisierungsversuche der Landarbeiter; Desinteresse der politischen Parteien an der politischen Klientel der Landarbeiter haben die Gründung von Gewerkschaften verhindert. 2) Ejidatarios leben nur zu einem Teil von ihrem Land (1/3 bis $1 / 2$ des Familieneinkommens); sie haben nur geringe Reserven (Kapital- und Immobilienbesitz), so dass der alltägliche Überlebenskampf ihre Energien bindet; Desinteresse der politischen Parteien und der formellen Bauernorganisationen.

34 Einige Frauen der Mittelschicht haben dann den Sprung in verantwortliche Positionen der Stadtpolitik (Stadtrat) geschafft. 


\section{Neues Verhältnis von Staat und Gesellschaft}

Die neoliberalen Politikreformen auf dem Land haben den mexikanischen Staat vor die Herausforderung gestellt, zu jedem Agrarproduzenten eine formale Beziehung aufzunehmen. Die allgemein vertretene Ansicht, dass der neoliberale Staat einer ist, der sich von der Landwirtschaft zurückgezogen hat, wird hier nicht in dieser Eindeutigkeit bestätigt. Im Gegenteil sehe ich die Hypothese von Jonathan Fox (1995: 1-30) bestätigt, dass die staatliche Intervention in der Landwirtschaft weitergeht, unter veränderten Vorzeichen zwar, aber in mancher Hinsicht tiefer eingreifend als je zuvor. Dies liegt einerseits daran, dass die erfolgreiche Implementierung der Pro-Markt-Reformen der Institutionen des Staates bedarf. Andererseits tritt hier die Glokalisierung als Effekt der „Vor-Ort-angekommenen“ Globalisierung zutage. Die These von Theodore J. Lowi (1992: 1-7) vom Markt als neuer „Sprache des Staates“ wird dadurch plausibel. Fox (1995: 1-30) erinnert daran, dass die klassische Variante der Intervention des mexikanischen Staats auf dem Land größtenteils indirekt war. In Fragen der Landwirtschaft legte die nationale Regierung einen großen Teil der staatlichen Autorität in lokale Hände. Die lokalen Vermittlungsinstitutionen (Ejido, Kaziquen, Landbesitzervereine) genossen relative Autonomie, ihre willkürliche Machtausübung bescherte dem politischen System langjährige Stabilität. Die staatliche Intervention auf den landwirtschaftlichen Märkten geschah ebenfalls hauptsächlich über Preisregulierung und öffentliche Investitionen. Für diese Art der staatlichen Intervention auf dem Land war keine institutionelle Beziehung zwischen dem Staat und dem individuellen Agrarproduzenten notwendig. Insbesondere in abgelegenen Regionen war der mexikanische Staat etwas, das der lokale Bauer nur vom Hörensagen kannte. Seine konkrete Erfahrung mit staatlicher Autorität machte er mit anonymen Preismechanismen und korrupten/autoritären Bossen. Mit der durchgreifenden Umsetzung der neoliberalen Landwirtschaftspolitik ab 1989 setzt hier eine Wende ein. PRONASOL entwickelt sich zum institutionellen Rahmen für Verhandlungen zwischen Staat und Landbevölkerung/Bauern. 1993 wurde PROCAMPO als Trostpflaster für die „sozialen Härten“ der Weltmarktöffnung eingeführt. Es sollte auch die politischen Kosten minimieren. Dieses sozialstaatliche Politikinstrument stellt hohe Anforderung an die Leistungsfähigkeit der staatlichen Institutionen, die plötzlich eine ungeheure Menge an präzisen Daten über die Landwirtschaft und die Bauern erheben und verwalten sollen. Wie der Fall Sayula bestätigt, tauchen bei der Erstellung eines vollständigen Agrarproduzentenzensus gleich drei Probleme auf: 1) legitime Produzenten können ausgelassen werden (z.B. wegen eingeschränkter Arbeitskapazität der personell unterausgestatteten Agrarbehörden oder aus betrügerischer Absicht); 2) illegitime Produzenten können fälschlicherweise in 
die Liste aufgenommen werden; 3) die Aufnahme in die Liste kann mit „politischen oder ökonomischen Bedingungen“" versehen sein (Unterordnung unter die PRI; Zahlung von Schmiergeldern). Eine systematische Dokumentation der Praxis der „politischen Verwendung" von Procampo und Procede gibt es (bislang) nicht. Auch im Fall von ProcedE soll der Staat in Gestalt des Agrarministeriums innerhalb von extrem kurzer Zeit verläßliche Daten über die Ejidos erheben, auf deren Basis das Privatisierungsprogramm durchgeführt wird. Die beiden Programme sind insofern auch politisch miteinander verbunden, als der PROCAMPO-Scheck an den PROCEDE-Titel gebunden ist. ${ }^{35}$ Obwohl die beiden Programme als neoliberale Elemente der staatlichen Landwirtschaftspolitik den Rückzug des Staates von der Landwirtschaft markieren sollen (als Effekt der Globalisierung), geschieht bei der Umsetzung vor Ort (Glokalisierung) genau das Gegenteil.

\section{Mexiko auf dem Weg zur Demokratie?}

Mexiko gehört in die wachsende Kategorie der „halbdemokratischen“ Regime (Fox 1994): in der politischen Sphäre (who governs and how?) gelten die demokratischen Normen des Parteienwettbewerbs und der Partizipation der $\mathrm{Zi}-$ vilgesellschaft (wenn auch nicht durchgängig und vollständig); in der ökonomischen Sphäre (who gets what and how?) setzt sich die staatliche Exekutive mit ihren sozioökonomischen Investitions- und Regulierungskriterien durch. Eine scharfe Trennlinie zwischen den beiden Aktionsfeldern des Staates gibt es nicht. Gerade auf dem Land kombinieren autoritäre Staatsparteien die Peitsche der begrenzten politischen Freiheiten mit dem Zuckerbrot der klientelistischen Anreize. Der Fall Sayula zeigt, dass nicht alle Bedingungen der politischen Demokratie erfüllt sind und daher von Demokratie nicht die Rede sein kann. Im Falle der PROCEDE-geschädigten Ejidatarios sehen wir, dass „das Recht auf Vereinigungsfreiheit nicht automatisch aus dem Machtwechsel auf nationaler (regionaler, lokaler, S.H.) Ebene folgt“ (Fox 1994: 151-184). Der durch Wahlen herbeigeführte Machtwechsel im Rathaus von Sayula, im Kongress von Jalisco oder auch im Nationalkongress garantiert noch nicht automatisch die demokratischen Grundrechte für die gesamte Bevölkerung. Dies liegt daran, dass das demokratische Grundrecht der Vereinigungs- und Versammlungsfreiheit ein politisch konstruiertes ist, an dem drei, miteinander im Konflikt liegende Schlüsselakteure arbeiten: 1) autonome soziale Bewegungen; 2) autoritäre Staatseliten, die am Machtmonopol festhalten wollen; und 3) reformistische Staatsagenten. Im Fall Sayula hat sich der Reformflügel von der PRI abgespalten und ist teilweise zu den anderen Parteien übergelaufen. Übrig blieben in der PRI die autoritären Hardliner, die aus ihren Schutz- und Trutzburgen - CNC und Agrarbehörden - ihre Fangarme ins Ejido ausstreck-

35 Diese Konditionalität wird von den Agrarbehörden geleugnet. 
ten. Der Machtwechsel im Rathaus hat an dieser Konstellation nichts geändert. Im Municipio Sayula wird auch deutlich, dass die staatliche Achtung der Menschenrechte nicht nur von Rasse und Region (Fox 1994: 157, Fußnote 18), sondern auch von der Klassenzugehörigkeit abhängt. Die lokalen Barzonisten, reiche Großbauern, haben spektakuläre Proteste organisiert, Gesetze übertreten, die Macht provoziert und zivilen Ungehorsam geleistet, sie haben den lokalen PRIistas die Macht streitig gemacht und sind schließlich ins Rathaus eingezogen. Es kam weder zu Verhaftungen, noch Klagen vor Gericht oder Einschüchterungsversuchen. Die PROCEDE-geschädigten Ejidatarios, allesamt Kleinbauern der unteren sozialen Schicht, haben längst nicht soviel politischen Staub aufgewirbelt, aber die Repression schlug brutal zu. Die autoritäre Elite zeigt damit, dass sie eher bereit ist in die parlamentarische Opposition zu gehen, als sich das verbliebene „domaine réservé", nämlich das Ejido, streitig machen zu lassen. Es geht nämlich nicht nur um politische Einflußnahme und persönliche Machtallüren, sondern um viel Geld und Land. Wenn der Begriff des „Semi-Klientelismus“ als sinnvolle Kategorie zur Erforschung der Beziehungen zwischen Staat und Gesellschaft im „halbdemokratischen " Transitionsregime Mexikos auf die mittlere und oberen Gesellschaftsschichten angewandt werden kann, da „die semiklientelistischen Machtbeziehungen eher durch die Vorenthaltung von Belohnung (carrots) als durch die Anwendung von Strafe (sticks) für Gehorsam sorgen“ (Fox 1994: 157), dann gilt dies nicht für die untere soziale Schicht. Im Fall Sayula sehen wir die alte Version des autoritären und repressiven Klientelismus am Werk. Auf Seiten der Adressaten geht es um den Unterschied zwischen Leben und Tod.

Das klassische Verständnis von Demokratisierung als einem einheitlichen Transformationsprozess des ganzen politischen Systems sollte daher neu gefasst werden als eine zusammenhängende Kette von Transformationselementen, die alle zusammen Kernelemente und Grundvoraussetzung für Demokratie sind. Es käme darauf an die Beziehungen zwischen den verschiedenen „genres of transition“ (Fox 1994) näher zu untersuchen. Die verschiedenen Aspekte der Transformation stehen in einer Wechselbeziehung zueinander und überschneiden sich in der Realität oft, aber logisch und historisch ${ }^{36}$ sind sie zu unterscheiden.

\section{Literatur}

36 Dies trifft insbesondere auf den schon erwähnten Faktor des Rassismus und der daraus folgenden Klassenverachtung zu. Die historische Erfahrung von 500 Jahre conquista hat ihre Spuren hinterlassen. 
Arroyo Alejandre, Jesús (comp.) (1995): $Y$ ante todo la población rural persiste. Efectos de la modernización económica en el campo mexicano, Guadalajara.

Calva, José Luis (1994): „En el lecho de muerte de la reforma agraria. Las reformas a la Ley Agraria”, S.7-32, in: José Luis Calva (ed.) et al., Linderos de la Reforma Agraria, Cuadernos de Investigación, UNAM, México.

Calva, José Luis et al. (1990): El ejido en México: crisis y modernización, Documento de trabajo No.29, Friedrich-Ebert Stiftung, México.

Cornelius, Wayne A./Ann L. Craig/Jonathan Fox (ed.) (1994): Transforming State-Society Relations in Mexico. The National Solidarity Strategy, U.S.-Mexico Contemporary Perspectives Series, No.6, La Jolla, Center of U.S.-Mexico Studies, University of California, San Diego.

Cuadernos Agrarios (1995): Neoliberalismo y campo, Nueva Epoca año 5, No. 11-12.

de Grammont, Hubert C./ Héctor Tejera Gaona (coord. generales) (1996): La Sociedad rural mexicana frente al nuevo mileno: Volumen I: La inserción de la agricultura mexicana en la economía mundial; Volumen II: La nueva relación campo-ciudad y la pobreza rural; Volumen III: El acceso a los recursos naturales y el desarollo sustentable; Volumen IV: Los nuevos actores sociales y procesos políticos en el campo, México.

De Grammont, Hubert C.: El Barzón cun movimiento social en contra de la crisis económica o un movimiento social de nuevo cuño?, Vortrag auf dem II Congreso de la Red de Estudios Rurales, Querétaro, 1.-4. März 1998.

DeWalt, Billie/Martha W.Rees (1994): The End of Agrarian Reform in Mexico. Past Lessons, Furture Prospects, Transformation of Rural Mexico No. 3, Ejido Reform Research Project, Center of U.S.-Mexican Studies, University of California, San Diego.

Encinas R., Alejandro/Juan de la Fuente/Horacio Mackinlay/Emilio Romero (comp.) (1995): El campo mexicano en el umbral del siglo XXI, México.

Friedmann, Harriet/Philip McMichael (1989): „Agriculture and the state system: the rise and decline of national agricultures, 1870 to present”, in: Sociologia Ruralis, No.29, pp. 93-117.

Gómez Cruz, Manuel Angel/Felipe de Jesús Caraveo (1990): „La agromaquila hortícola: nueva forma de penetración de las transnacionales”, in: Comercio Exterior, vol. 40, No.12, México.

Léonard, Eric: Las reformas estructurales y su impacto sobre la movilidad social en las agriculturas ejidales de la sierra de Los Tuxtlas, Veracruz, Vortrag auf dem II Congreso de la Red de Estudios Rurales, Querétaro, 1.-4. März 1998.

Lowi, Theodore (1992): „The State in Political Science: How We Become What We Study”, in: American Political Science Review 86(1).

Meyer, Jean (1973): La révolution mexicaine, Paris.

Ocampo Alcántar, Rigoberto (comp.) (1992): Teoría del Neocorporatismo. Ensayos de Philippe C. Schmitter, Guadalajara.

Orozco Alvarado, Javier/Ricardo Fletes Corona (coord.) (1996): Estratégias regionales y nacionales frente a la integración económica mundial, El Colegio de Jalisco, México.

Paré, Luisa (1990): „The Challenges of Rural Democratization in Mexico”, S.79-96, in: Jonathan Fox (ed.), The Challenge of Rural Democratization: Perspectives from Latin America and the Philippines, London.

Ross, John (1997): „Zapata's Children. Defending the Land and Human Rights in the Countryside", in: NACLA; Vol. XXX, No.4, p. 30-35.

Stephen, Lynn (1994): Viva Zapata! Generation, Gender and Historical Consciousness in the Reception of Ejido Reform in Oaxaca, Transformation of Rural Mexico No. 6, Ejido Reform Research Project, Center of U.S.-Mexican Studies, University of California, San Diego.

Torres, Gabriel: El Barzón del agave: Plusvalia y Sobreproducción en un contexto de globalización, Vortrag auf dem II Congreso de la Red de Estudios Rurales, Querétaro, 1.4. März 1988. 